\title{
Friction of Polymers Sliding on Smooth Surfaces
}

\author{
Virginio Quaglini and Paolo Dubini \\ Dipartimento di Ingegneria Strutturale, Politecnico di Milano, Piazza Leonardo da Vinci 32, 20133 Milano, Italy \\ Correspondence should be addressed to Virginio Quaglini, virginio.quaglini@polimi.it
}

Received 24 June 2011; Revised 9 September 2011; Accepted 20 September 2011

Academic Editor: Shyam Bahadur

Copyright ( 2011 V. Quaglini and P. Dubini. This is an open access article distributed under the Creative Commons Attribution License, which permits unrestricted use, distribution, and reproduction in any medium, provided the original work is properly cited.

Friction plots of polymers sliding on smooth metal surfaces are generally characterized by two regions of distinct dependency on the normal load, with low sensitivity at low stress levels followed by a sharp change in the rate of decrease of friction with increasing pressure at levels above the plastic flow limit of the polymer. A simplified model is proposed to describe this behavior which accounts for the effect of the normal load on the growth of the real contact area and the shear stress at the interface between the polymer and the mating surface. The model has a wide generality when expressed in terms of dimensionless variables, allowing to rationalize the friction behavior of different polymers within a single framework.

\section{Introduction}

Sliding bearings incorporating self-lubricating polymers are today widely employed in several fields of engineering, including mechanical, structural, civil, and biomedical, for their ability to provide low friction without the need of external lubrication, their low cost, and minimum maintenance burden. In such bearings the sliding surface of the polymer element usually matches a partner surface made of steel or other hard metals, since polymers are known to be more effective, as concerns friction and wear performances, against a metal than when sliding against themselves [1].

To achieve correct design and selection of the bearings according to the intended service conditions, assessing the influence of operating parameters like normal load, temperature, and velocity on the coefficient of friction of the polymer is crucial. To this aim, both semiempirical laws and physically sound models involving a large number of parameters have been developed. The sliding behavior of polymers has been widely studied over the last 50 years, and a number of wellestablished models of friction are today available (a recent review is reported in Myshkin and Petrokovets [2]).

Two main mechanisms are acknowledged to contribute to the friction force between a polymer pad and a metal surface: the shearing of the junctions formed by adhesion between the asperities of the contacting surfaces, and the dissipation of energy due to and the dissipation of energy due to plastic deformation and abrasion $[1,3,4]$ : under high contact stresses the asperities of the harder material plough the softer one, and the resistance to ploughing further contributes to increase the frictional force [5].

However, it is generally accepted that at low and medium pressure levels ploughing of a polymer on smooth metal surfaces can be neglected [6-8], and friction is explained in terms of the adhesion mechanism only, as firstly proposed by Shooter and Tabor [9].

When a polymer pad is rubbed against a steel plate under the normal load $F_{N}$, adhesive junctions form at the regions of true contact between the mating surfaces, and the shear force that resists mutual sliding is given by

$$
F_{T}=\tau_{s} \cdot A_{r},
$$

where $A_{r}$ is the real contact area of the junctions and $\tau_{s}$ is the shear stress required to produce sliding between the surfaces. As the shear force $F$ is less than $F_{T}$, motion between the surfaces is prevented, while relative sliding occurs under $F=F_{T}$.

The value of $\tau_{s}$ in (1) coincides with the lowest between the strength of adhesion at the interface $\left(\tau_{i}\right)$ between the asperities $[10,11]$ and the bulk shear strength of the polymer $\left(\tau_{b}\right)$. If $\tau_{i}<\tau_{b}$, then sliding occurs truly at the interface; if $\tau_{i}>\tau_{b}$, shearing, followed by detachment of a polymer 
fragment, will take place in the bulk of the polymer at a short distance from the interface [12]. For thermoplastic polymers these strength properties are generally of the same order of magnitude, as most polymers adhere to other materials by van der Waals forces and appear to vary with temperature, especially near the glass transition temperature.

Another key element to be considered regarding the friction of polymers is the influence of the normal force on the real contact area, through the mechanism of junction growth [3]. Both theoretical and experimental studies [3, 1315] concluded that, either in the case of purely elastic or elastoplastic deformation of the asperities, the real area of contact is almost linearly proportional to the load, as $A_{r}=k$. $\left(F_{N}\right)^{m}$ with $m$ close to unity. Starting from (1) and neglecting the contribution of plastic dissipation, the coefficient of friction $\mu$ of a polymer sliding against a smooth surface can be expressed as

$$
\mu=\frac{F_{T}}{F_{N}}=k \cdot \tau_{s} \cdot\left(F_{N}\right)^{m-1},
$$

where the factor of proportionality $k$ depends on several factors including the shape and distribution of the asperities and the bulk properties of the polymer.

If $m=1$, then $\mu$ is a a constant, according to the Coulomb model of friction, which is the case, for example, of metals. Equation (2) proved to fit experimental data for several thermoplastic polymers with values of $m$ between 0.74 and $0.96[16,17]$, though the exponent $m$ was found to be not constant over large ranges of load, with significant deviations of the polymer behavior from the model [8].

In the present investigation the above theory is refined, based upon particular assumptions on the dependency of the real contact area $A_{r}$ and the limit shear stress $\tau_{s}$ on the normal load, and a simple model of adhesive friction for polymers is derived. The physical consistency of the model is then assessed upon experimental friction-pressure data for some among the most common polymers currently used in sliding bearing manufacturing.

\section{Experiments}

2.1. Materials and Procedure. The experimental investigation was carried out on five self-lubricating polymers: Polyethylene terephtalate (PET), cast Polyamide 6 (PA6), Polyvinylidene fluoride (PVDF), ultrahigh-molecular-weight polyethylene (UHMWPE), and Polytetrafluorethylene (PTFE).

Friction tests were conducted using circular specimens of the self-lubricating polymers, rubbed against a partner sheet made of stainless steel, type AISI 316, with surface finish to $\mathrm{Ra} \leq 0.03 \mu \mathrm{m}$ and $\mathrm{Rz} \leq 0.12 \mu \mathrm{m}$ in order to minimize the ploughing effect.

The diameter of the polymer specimen was $25 \mathrm{~mm}$ for the harder materials (PA6, PVDF, and PET) in order to allow the application of higher specific loads and $65 \mathrm{~mm}$ for the softer materials (PTFE and UHMWPE), resulting in better accuracy in measurement of the friction force especially at low pressure levels.

The specimens were placed in a custom designed testing machine, as sketched in Figure 1. The machine consists of

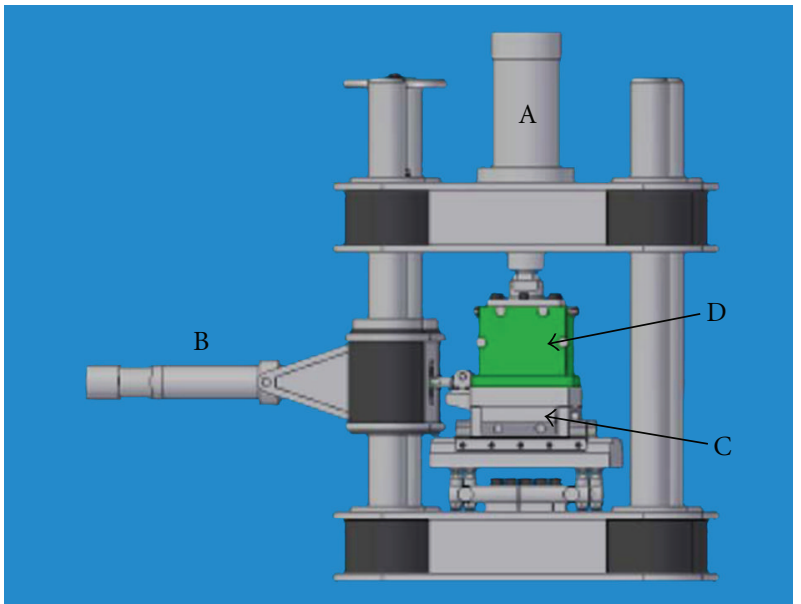

FIgure 1: Schematic drawing of the testing machine. (A) Vertical jack for application of the compressive load; (B) horizontal jack for application of sliding movement; (C) sledge supported by roller bearings; (D) thermal chamber. The specimen (not visible) supported by the sledge (C) is enclosed in the thermal chamber (D) during the test.

a four-column steel frame equipped with two servohydraulic jacks. The vertical jack (item (A) in Figure 1), with capacity of $300 \mathrm{kN}$, applies a compressive load to the specimen as to produce the rated pressure level. The horizontal jack (item (B)), with capacity of $50 \mathrm{kN}$, drives a sledge (item (C)) moving with negligible friction on a set of roller bearings. The specimen of self-lubricating material is carried by the sledge and slides onto the partner steel sheet that is supported by a backing plate on the rod of the vertical jack (item (A)). Load cells and inductive displacement transducers are used to measure the loads and the movements applied by the jacks at the sliding interface of the specimen. Both jacks are driven in closed-loop by servovalves to ensure accurate control of load and velocity.

The polymer specimen and the steel sheet are confined in a thermal chamber (item (D)) installed on the testing machine which operates between $-70^{\circ} \mathrm{C}$ and $90^{\circ} \mathrm{C}$ within a tolerance of $\pm 1^{\circ} \mathrm{C}$. During the tests the temperature was set to $21^{\circ} \pm 1^{\circ} \mathrm{C}$.

The coefficient of friction of each polymer was measured at various levels of normal load $F_{N}$. At each level, the load was applied to the specimen and held constant for 30 minutes, in order to allow for viscoelastic deformation of the polymer asperities to occur, and then one movement was performed at the constant speed of $1 \mathrm{~mm} / \mathrm{s}$. At the end of the movement, the actual diameter of the polymer pad and the roughness of the sliding surfaces were measured. The current diameter, $d$, was used to calculate the nominal area of contact $A_{n}=$ $\pi \cdot d^{2} / 4$, and hence the nominal contact pressure $p_{n}=$ $F_{N} / A_{n}$ at each load level. The contribution of ploughing and deformation to friction was assumed to be negligible (lower than 0.002) if the roughness of the steel plate did not exceed $\mathrm{Ra}=0.06 \mu \mathrm{m}$, otherwise the friction data taken at that level were discharged and the test stopped. 
TABLE 1: Hardness of the tested polymers measured according to ISO 2039-1 ball indentation method for plastics.

\begin{tabular}{lccccc}
\hline & PTFE & UHMWPE & PET & PVDF & PA6 \\
\hline$H\left[\mathrm{~N} / \mathrm{mm}^{2}\right]$ & 27 & 25 & 94 & 74 & 88 \\
\hline
\end{tabular}

2.2. Test Results. The results are illustrated in the friction plots of Figure 2, which show the dynamic coefficient of friction $\mu$ as a function of the nominal pressure $p_{n}$ at temperature of $21^{\circ} \mathrm{C}$ and velocity of $1 \mathrm{~mm} / \mathrm{s}$.

All polymers were tested over a very large range of pressures, from a few MPa to levels well above their yield stress. As expected, PTFE has the lowest friction resistance, while PA6, PET, and PVDF exhibit friction values more than three times larged than those of PTFE. The coefficient of friction of UHMWPE has intermediate values.

Though the friction coefficients of the materials are well distinct, all polymers exhibit a similar behavior, with a change in the friction force less than proportional to the applied load, resulting in a decrease of the coefficient of friction as pressure increases. However, differently from what expected according to (2), the relationship between friction coefficient and pressure is characterized by two regions of different sensitivity: at high loads, the coefficient of friction has a faster decrease as the normal load is increased, while in the range of low and moderate loads the coefficient of friction changes more slowly, or even holds constant. The transition between these two regions occurs in a narrow range of pressures that is characteristic of each polymer and seems to be related to the material hardness (Table 1): the transition takes place in the range 25 to $35 \mathrm{MPa}$ for the softer polymers (PTFE and UHMWPE) and at above $60 \mathrm{MPa}$ for the harder ones.

\section{Adhesion and Friction}

3.1. Model Development. The frictional resistance of a polymer pad sliding on a smooth metal surface, like in the experiments described in Section 2, can be considered as the effect of the adhesive contribution only. The true contact between the two bodies generally occurs at a limited number of regions of the mating asperities, and the real contact area $A_{r}$ is lower than the apparent or nominal contact area $A_{n}$ between the surfaces. When the normal load $F_{N}$ only is acting, each asperity may experience either elastic or plastic deformation depending on whether the local pressure is smaller or greater than the plastic flow stress (yield stress) of the polymer, $\sigma_{Y}$.

However, when the contacting surfaces slide, the shear stresses favour the plastic flow of each asperity and the onset of plastic deformation at the contacting asperities is controlled by the combined effect of the normal stress $p=$ $F_{N} / A_{r}$ and shear stress $\tau=F_{T} / A_{r}$, according to the following relation:

$$
\left(\frac{F_{N}}{A_{r}}\right)^{2}+C \cdot\left(\frac{F_{T}}{A_{r}}\right)^{2}=\sigma_{Y}^{2}
$$

Here, $C$ is a constitutive parameter that depends on the material properties at the asperities [18-20]. Since the shear force at the contacting points is related to normal load by the coefficient of friction, $\mu=F_{T} / F_{N}$, substituting into (3) gives the limit normal load which produces plastic deformation of the polymer at the junctions during sliding:

$$
F_{Y}^{\prime}=\frac{\sigma_{Y} \cdot A_{r}}{\sqrt{1+C \cdot \mu^{2}}}
$$

According to the theory of junction growth [3], the real contact area $A_{r}$ depends on the normal load.

At low values, for $F_{N}<F_{Y}^{\prime}$ the asperities deform elastically and the effect of increasing the load is to increase the elastic deformation of each asperity. As discussed earlier, several investigations confirmed that in this situation the dependency of $A_{r}$ on $F_{N}$ is almost proportional.

As $F_{N}$ approaches $F_{Y}^{\prime}$, the asperities start to deform plastically; a further increase in the normal load will produce a flattening of the deformed asperities, new asperities come in contact, and the real contact area $A_{r}$ increases again proportionally with $F_{N}$.

Finally once the real contact area equals the nominal area of contact $A_{n}$ between the partner surfaces, which occurs at the characteristic value of normal load

$$
F_{Y}=\frac{\sigma_{Y} \cdot A_{n}}{\sqrt{1+C \cdot \mu^{2}}}
$$

$A_{r}$ becomes stable and a further increase in $F_{N}$ will not produce any increase in $A_{r}$.

The relationship between the real contact area $A_{r}$ and the normal load can hence be described as

$$
A_{r}= \begin{cases}k \cdot\left(F_{N}\right)^{m}, & \text { for } F_{N}<F_{Y} \\ A_{n}, & \text { for } F_{N} \geq F_{Y}\end{cases}
$$

where the exponential term $m$ is close to unity.

According to (1), the frictional force $F_{T}$ developed during sliding is given by the product of $A_{r}$ and $\tau_{s}$, where $\tau_{s}$ is the lowest between the bulk shear strength of the polymer and the adhesive strength at the interface. For polymers both strengths are generally of the same order of magnitude, and the two mechanisms of shearing can coexist at distinct junctions in the same polymer/metal pair.

Since the bulk shear strength of polymers is proportional to the pressure [21], while the interface adhesive strength is expected to be independent of the normal load [3], in the most general way the average shear strength of the junctions, $\tau_{s}$, can be assumed to be related to the real pressure of contact according to the law

$$
\tau_{s}=\tau_{0} \cdot\left(\frac{F_{N}}{A_{r}}\right)^{n}
$$

where $n$ is close to unity if shearing within the polymer prevails and close to zero in case of weaker adhesive forces at the interfaces.

Then rearranging the definition of friction coefficient according to (1), (6), and (7) and replacing $F_{N}$ by the 

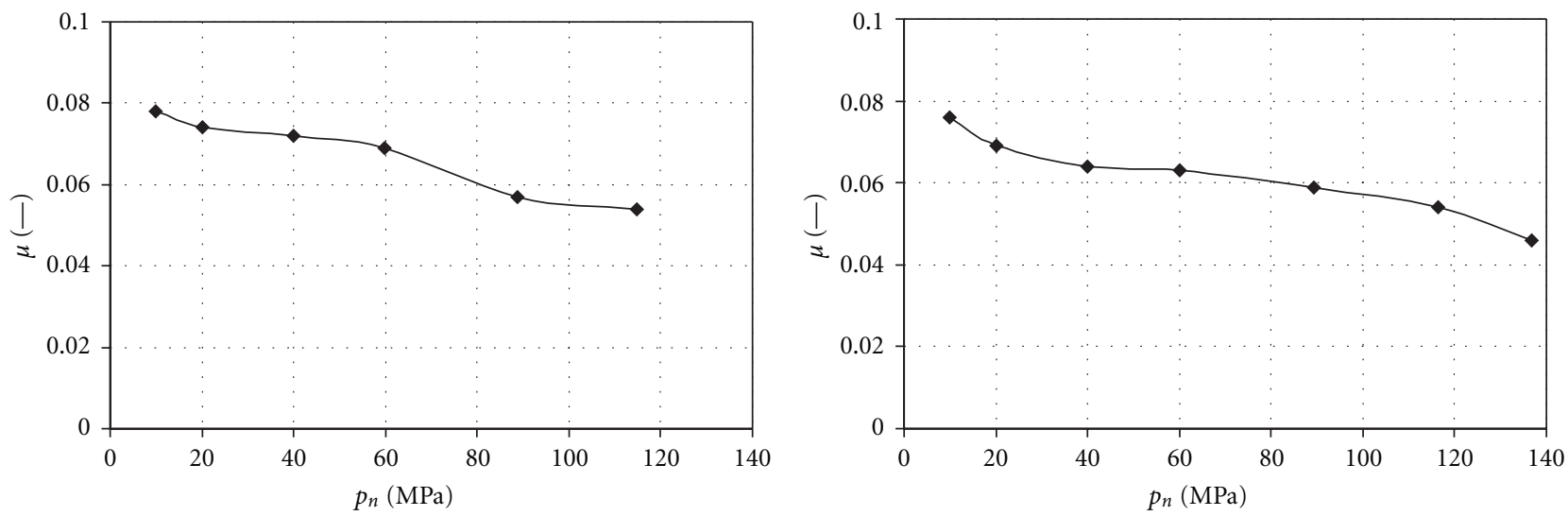
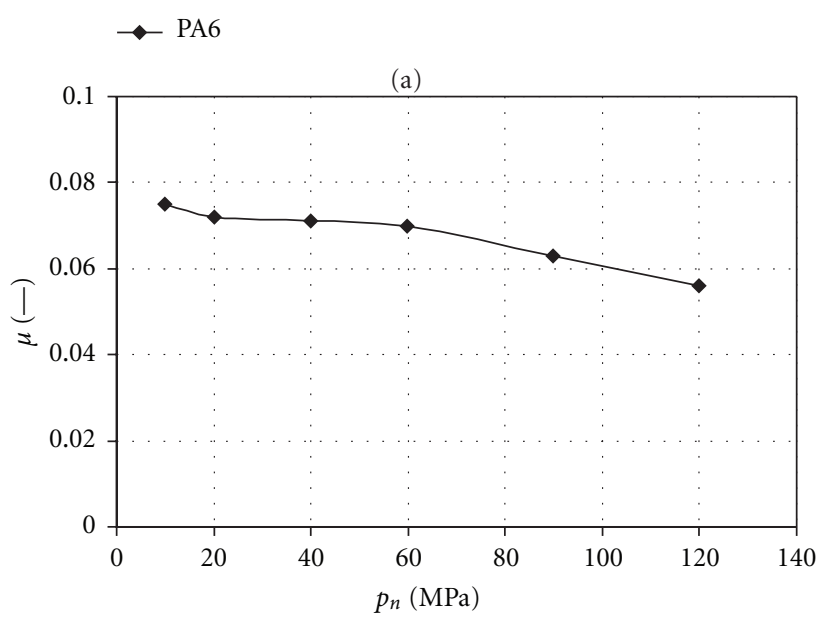

PET

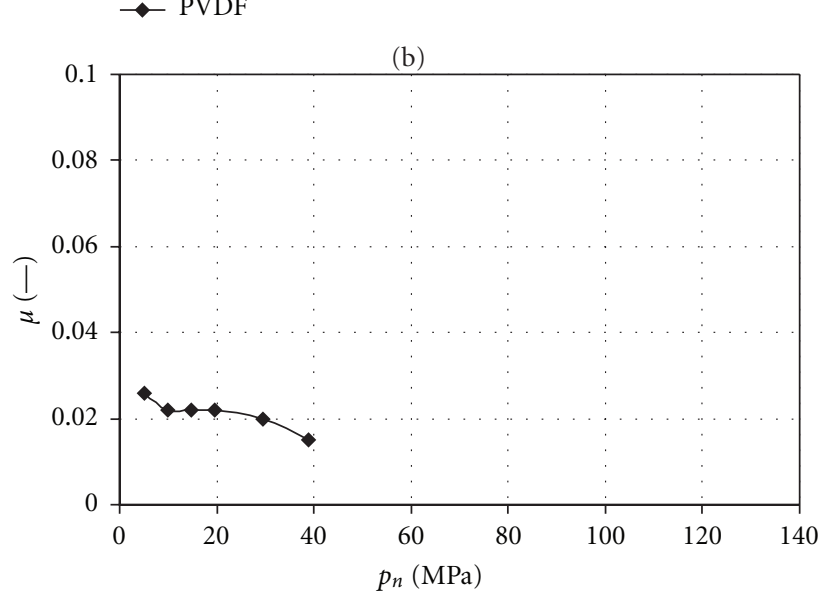

- PTFE

(c)

(d)

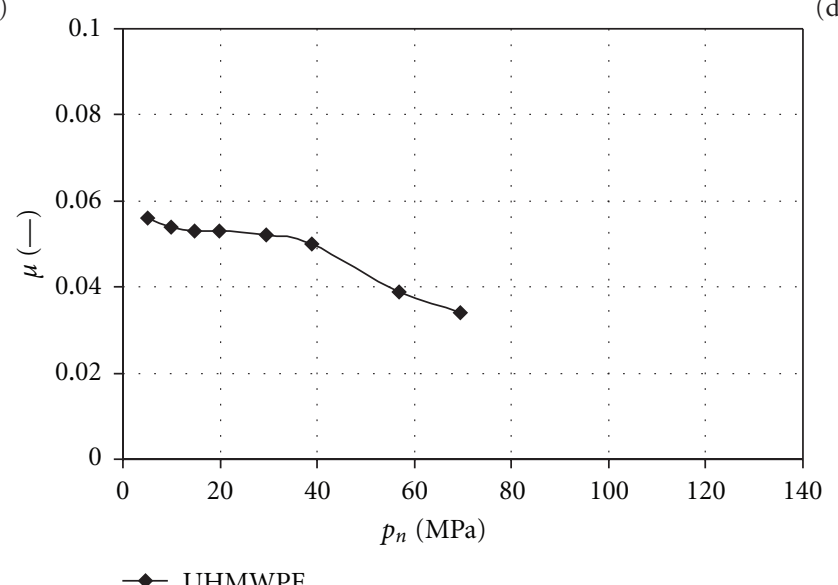

(e)

FIgURE 2: Experimental plots of the coefficient of friction $\mu$ versus nominal contact pressure $p_{n}=F_{N} / A_{n}$, for different polymers tested at $21^{\circ} \pm 1^{\circ} \mathrm{C}$ and $1 \mathrm{~mm} / \mathrm{s}$ velocity.

nominal pressure $p_{n}=F_{N} / A_{n}$, one finds the following relation:

$$
\mu= \begin{cases}\frac{k_{1}}{\left(p_{n}\right)^{\alpha}}, & \text { for } p_{n}<p_{Y}, \\ \frac{k_{2}}{\left(p_{n}\right)^{\beta}}, & \text { for } p_{n} \geq p_{Y} .\end{cases}
$$

Here, $p_{Y}=\sigma_{Y} / \sqrt{1+C \cdot\left(\mu_{P Y}\right)^{2}}$ is the transition pressure (where $\mu_{P Y}$ is the corresponding value of the dynamic coefficient of friction), $\alpha$ and $\beta$ are two parameters that depend on the bulk properties of the polymer (modulus of elasticity and shear strength) and on the adhesion force of the polymer against the mating surface, and $k_{1}$ and $k_{2}$ are two parameters accounting for the geometry of the asperities 


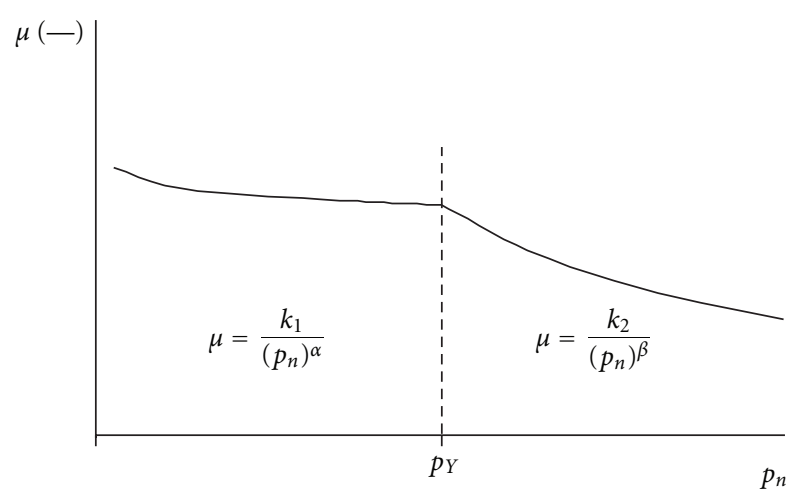

Figure 3: Coefficient of dynamic friction $\mu$ versus nominal contact pressure $p_{n}$ according to (8).

TABLE 2: Coefficients of the friction model. Equation (9): exponential terms $\alpha$ and $\beta$, and material properties $p_{Y}$ and $\mu_{P Y}$ of the tested polymers determined by curve fitting the dimensionless friction plots of Figure 4.

\begin{tabular}{lcccc}
\hline polymer & $\alpha[-]$ & $\beta[-]$ & $p_{Y}\left[\mathrm{~N} / \mathrm{mm}^{2}\right]$ & $\mu_{P Y}[-]$ \\
\hline PTFE & 0.06 & 0.90 & 27 & 0.021 \\
UHMWPE & 0.06 & 0.70 & 40 & 0.050 \\
PET & 0.09 & 0.44 & 80 & 0.066 \\
PVDF & 0.11 & 0.39 & 81 & 0.006 \\
PA6 & 0.12 & 0.50 & 70 & 0.065 \\
\hline
\end{tabular}

of the two surfaces. The coefficient of friction is explicitly related to the nominal pressure $p_{n}$, instead of to the normal load $F_{N}$, to account for plastic deformation of the polymer pad and the following increase in $A_{n}$ at high normal loads.

The bifurcation expression of (8) allows for two distinct regions of dependency of the friction coefficient on the contact pressure, depending on whether $p_{n}$ is above or below the plastic flow stress $p_{Y}$, as sketched in Figure 3. For $p_{n}<$ $p_{Y}$, the real contact area increases with increase of pressure; for $p_{n} \geq p_{Y}$, the real contact area and the nominal area do coincide; in the intermediate region the real contact area approaches the nominal area in a way that depends on the geometric characteristics of the surfaces and the modulus of elasticity of the mating materials.

3.2. Model Calibration. The model has wider generality if the dimensionless variables are introduced:

$$
\begin{aligned}
& \bar{p}=\frac{p_{n}}{p_{Y}}, \\
& \bar{\mu}=\frac{\mu}{\mu_{P Y}},
\end{aligned}
$$

where $\mu_{P Y}$ is the value of the dynamic coefficient of friction at the transition pressure $p_{n}=p_{Y}$.
With respect to the new variables $\bar{p}$ and $\bar{\mu}$, (8) takes the simpler expression

$$
\bar{\mu}= \begin{cases}\frac{1}{\bar{p}^{\alpha}}, & \text { for } \bar{p}<1, \\ \frac{1}{\bar{p}^{\beta}}, & \text { for } \bar{p} \geq 1,\end{cases}
$$

where the dependency on the geometry of asperities expressed by parameters $k_{1}$ and $k_{2}$ is formally ruled out by the normalization procedure.

The consistency of the model with the actual behavior of self-lubricating polymers was assessed by curve fitting the normalized friction versus pressure plots of the materials tested in Section 2 with (10). The curve fitting is illustrated in Figure 4 and the estimated values of the parameters are given in Table 2. The RMS (root mean square) error between the experimental data and the model has been calculated for each material and reported in the relevant plot of Figure 4.

The agreement between experimental data and curve fitting was satisfactory for all polymers, with RMS errors ranging between a minimum of 0.005 for UHMWPE and a maximum of 0.015 for PA6. The largest deviations occurred at the lowest values of $\bar{p}$; however, this gap could be a consequence of larger signal-to-noise ratio in the measure of small frictional forces at low pressures rather than in a lower accuracy of the model. As the pressure level increases, the deviation between the experimental and the model data point becomes negligible, with relative deviations below $1 \%$. The accuracy of the model was virtually equivalent in both regions of different sensitivity of the friction versus pressure plots.

\section{Discussion}

Different thermoplastics, chosen among the most popular self-lubricating polymers used in current bearing technology, were tested and the friction coefficient was measured over extended ranges of normal load. The relevant friction versus pressure plots of all materials, though at different levels and characterized by distinct friction values, showed a similar behavior with a steep change in the slope beyond a transition value typical of each material.

A constitutive law was therefore developed to represent this particular behavior starting from the background of the theory of adhesive friction and introducing two main concepts:

(1) the growth of real contact area, due either to elastic or to plastic deformation of asperities, occurs as far as the nominal pressure is below a threshold value, corresponding to plastic deformation of the whole polymer surface;

(2) the limit shear stress $\tau_{s}$ depends on two different mechanisms that can be active at different junctions.

The lower sensitivity of the friction coefficient on the normal load at low and moderate levels of pressure is explained as an effect of the growth of the real contact area by elastoplastic 


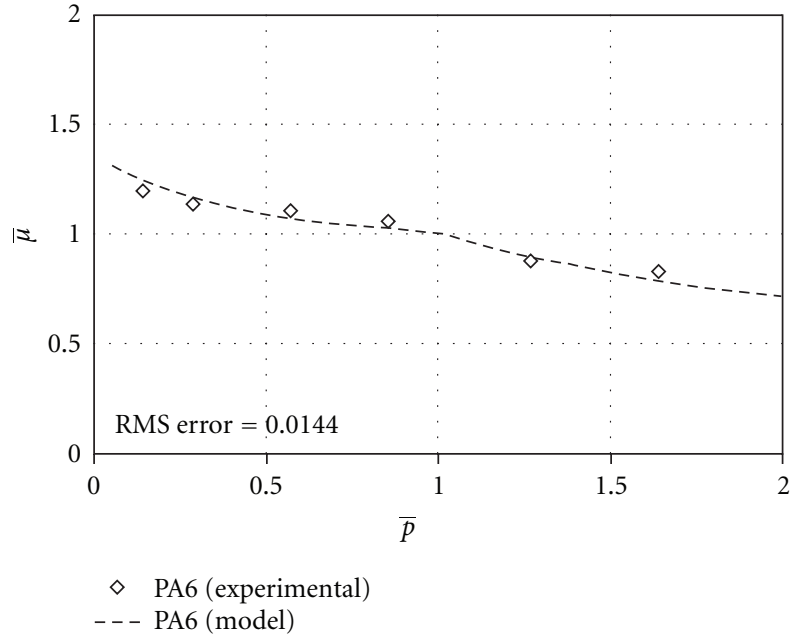

(a)

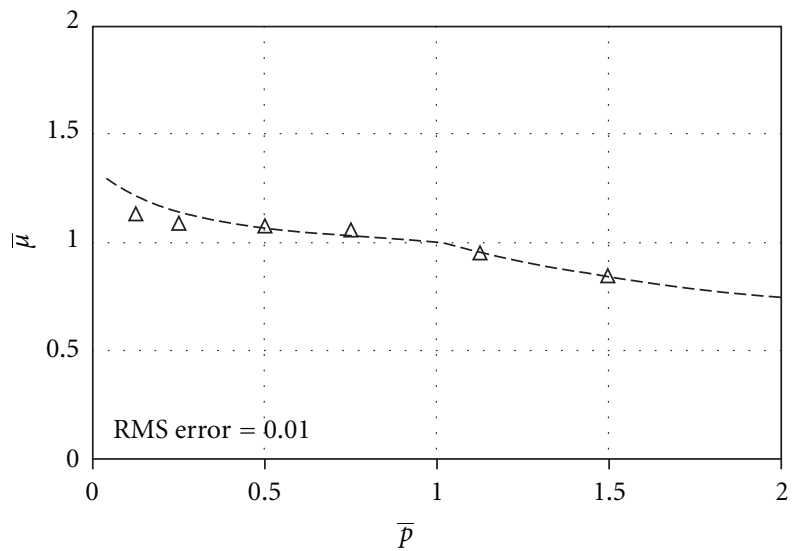

$\triangle$ PET (experimental)

- - - PET (model)

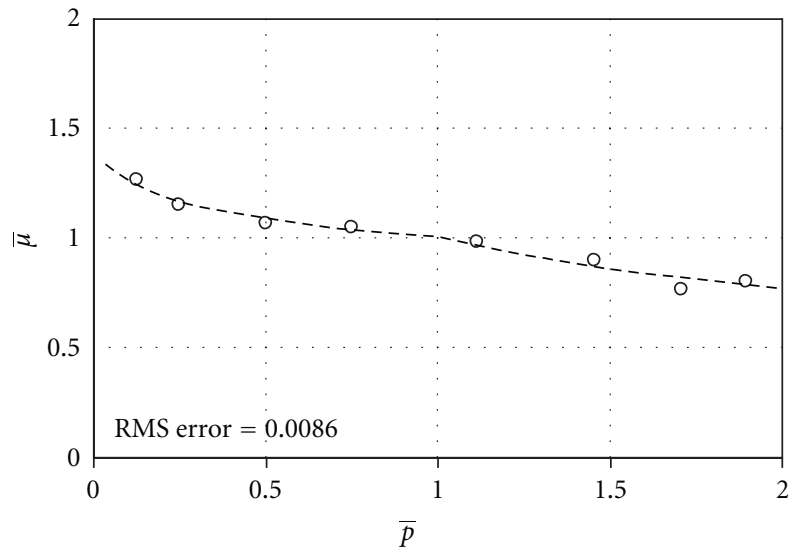

- PVDF (experimental)

- - - PVDF (model)

(b)

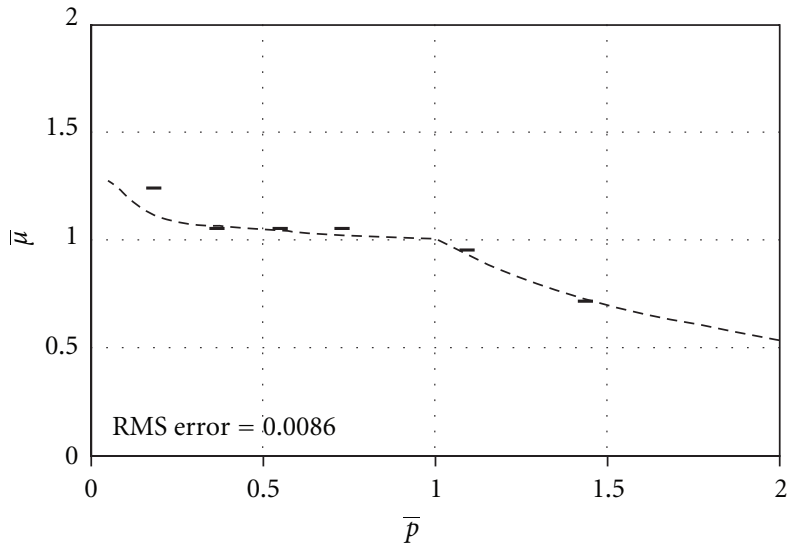

- PTFE (experimental)

- - - PTFE (model)

(c)

(d)

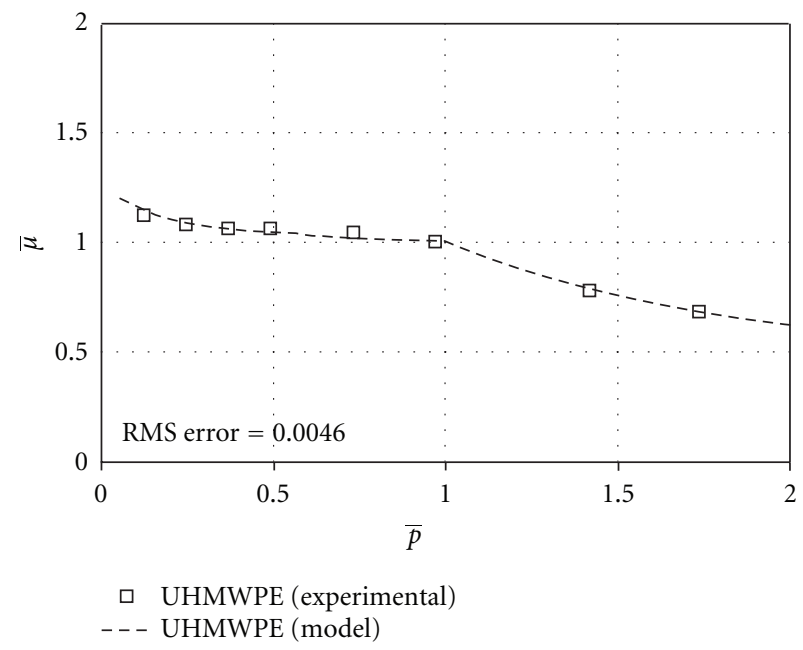

(e)

Figure 4: Curve fitting of experimental friction curves by the friction law of (10) with respect to dimensionless variables $\bar{p}$ and $\bar{\mu}$ defined in (9) and relevant root mean square (RMS) error. Model parameters are given in Table 2. 
deformation of the asperities, which is nearly proportional to the load. The faster rate of decrease of friction occurring at high levels of pressure, larger than the plastic flow stress of the polymer $p_{Y}$, is justified assuming that now contact occurs on the whole surface of the polymer.

The model shows a wider generality when it is expressed, like (10), in terms of the dimensionless variables (9), allowing to rationalize the friction characteristics of different polymers within a single framework.

Although very simple in its form, the model fits well the experimental data of different polymers, characterized by distinct friction coefficients, in a wide range of $p / p_{Y}$ values (from 0.025 to about 2.0), covering a range of material properties and friction behaviors.

The properties of each material within the model are described by four constitutive parameters, $p_{Y}, \mu_{P Y}, \alpha$, and $\beta$. A physical interpretation can be attached to each parameter.

The coordinates of the transition point between the two regions of the $\bar{\mu}-\bar{p}$ plot with different slope are related to the bulk properties of the polymer: $p_{Y}$ is the plastic flow stress in presence of shear stress due to sliding, and $\mu_{P Y}$ is the corresponding value of coefficient of friction. These parameters are related to the yield stress $\sigma_{Y}$ and to the parameter $C$ according to $p_{Y}=\sigma_{Y} / \sqrt{1+C \cdot\left(\mu_{P Y}\right)^{2}}$.

Comparison of values of $p_{Y}$ determined from curve fitting (Table 2) and hardness values of the tested materials (Table 1) shows that the estimated plastic flow stress is proportional to the hardness number $H$ of the polymer itself, consistently with the well-known proportionality between the yield stress $\sigma_{Y}$ and $H$ [21].

The power coefficient $\alpha$ in (10) accounts for the growth of the real contact area with the normal load due to the deformation of the asperities. Values of $\alpha$ between 0.05 and 0.15 were determined for the various polymers, in agreement with theoretical models based on the assumption of multiple asperities contact $[3,13,15]$, which require $\alpha$ close to unity.

The power coefficient $\beta$ in (10) is related to the change in shear strength at the junctions with load: low values, close to zero, denote shear strength proportional to load, characteristic of a predominant mechanism of shearing occurring within the bulk of the polymer, while values of $\beta$ approaching unity correspond to constant shear strength and predominant mechanism of sliding at the interface. $\beta$ was evaluated to be about $0.4-0.5$ for PA6, PET, and PVDF and larger for UHMWPE $(\beta=0.7)$ and PTFE $(\beta=0.9)$. This latter behavior, that is, prevailing of sliding at the interface, is likely to be related to the creation of a transfer film of polymer on the mating surface, which is typical of PTFE and, at a small extension, of UHMWPE $[1,12]$. Since the intrinsic adhesion between two surfaces made of the same polymer is very low, the frictional resistance is governed by the weak adhesive strength of the interface.

\section{Conclusions}

In this paper, starting from the classical theory of adhesive friction, a novel model is developed and a constitutive law is derived to describe the nonproportional dependency of the coefficient of friction of polymers on the contact pressure.
The model proved to be able to fit experimental friction plots of different polymers and to account for the change in the slope of the plot beyond a transition value of pressure characteristic of each material.

The main advantages of the model are its simplicity, since the material properties are described by four variables only which can be determined by curve fitting experimental friction versus pressure plots, and its generality when it is expressed in terms of dimensionless variables, allowing to describe the friction behavior of different polymers within a single constitutive framework.

The model can be usefully employed to represent the frictional behavior of most self-lubricating polymers used in current bearing technology.

\section{Acknowledgment}

The research was carried out with the support of Fondazione Cariplo (Grant 2008.2295).

\section{References}

[1] G. W. Stachowiak and A. W. Batchelor, Engineering Tribology, Butteworth Heinemann, Melbourne, Australia, 2005.

[2] N. K. Myshkin and M. I. Petrokovets, "Mechanical behavior of plastics: surface properties and tribology," in Mechanical Tribology. Materials, Characterization, and Applications, G. E. Totten and H. Liang, Eds., Marcel Dekker, New York, NY, USA, 2004.

[3] F. P. Bowden and D. Tabor, Friction and Lubrication of Solids, Clarendon Press, Oxford, UK, 1964.

[4] V. A. Bely, A. I. Sviridenok, M. I. Petrokovets, and V. G. Savkin, Friction and Wear in Polymer-Based Materials, Pergamon Press, Oxford, UK, 1982.

[5] H. Unal and A. Mimaroglu, "Friction and wear behaviour of unfilled engineering thermoplastics," Materials and Design, vol. 24, no. 3, pp. 183-187, 2003.

[6] S. Bahadur, "The development of transfer layers and their role in polymer tribology," Wear, vol. 245, no. 1-2, pp. 92-99, 2000.

[7] J. Gao, S. Mao, J. Liu, and D. Feng, "Tribochemical effects of some polymers/stainless steel," Wear, vol. 212, no. 2, pp. 238243, 1997.

[8] V. Quaglini, P. Dubini, D. Ferroni, and C. Poggi, "Influence of counterface roughness on friction properties of engineering plastics for bearing applications," Materials and Design, vol. 30, no. 5, pp. 1650-1658, 2009.

[9] K. V. Shooter and D. Tabor, "The frictional properties of plastics," Proceedings of the Physical Society. Section B, vol. 65, no. 9, pp. 661-671, 1952.

[10] D. Landheer and A. W. J. de GeeMaruyama, "Adhesion, friction and wear," Marine Pollution Bulletin, vol. 16, pp. 3640, 1991.

[11] K. H. Zum Gahr, Microstructure and Wear of Materials, Elsevier, Amsterdam, The Netherlands, 1987.

[12] C. M. Pooley and D. Tabor, "Friction and molecular structure: the behavior of some thermoplastics," Proceedings of the Royal Society A, vol. 329, no. 1578, pp. 251-274, 1972.

[13] I. M. Hutchings, Tribology: Friction and Wear of Engineering Materials, Edward Arnold, London, UK, 1992.

[14] Y. Yamaguchi, Tribology of Plastic Materials, Elsevier, Amsterdam, The Netherland, 1990. 
[15] N. K. Myshkin, M. I. Petrokovets, and A. V. Kovalev, "Tribology of polymers: adhesion, friction, wear, and mass-transfer," Tribology International, vol. 38, no. 11-12, pp. 910-921, 2005.

[16] M. W. Pascoe and D. Tabor, "The friction and deformation of polymers," Proceedings of the Royal Society of London. Series A, vol. 235, no. 1201, pp. 210-224, 1956.

[17] H. G. Howell and J. Mazur, "Amontons' law and fibre friction," Journal of Textile Institution, vol. 44, pp. T59-T69, 1953.

[18] S. C. Lim and M. F. Ashby, "Overview no. 55 Wear-Mechanism maps," Acta Metallurgica, vol. 35, no. 1, pp. 1-24, 1987.

[19] F. P. Bowden and D. Tabor, "Friction, lubrication and wear: a survey of work during the last decade," British Journal of Applied Physics, vol. 17, no. 12, article no. 301, pp. 1521-1544, 1966.

[20] K. C. Ludema, Friction, Wear, Lubrication: A Textbook in Tribology, CRC Press, New York, NY, USA, 1996.

[21] R. C. Bowers, "Coefficient of friction of high polymers as a function of pressure," Journal of Applied Physics, vol. 42, no. 12, pp. 4961-4970, 1971. 

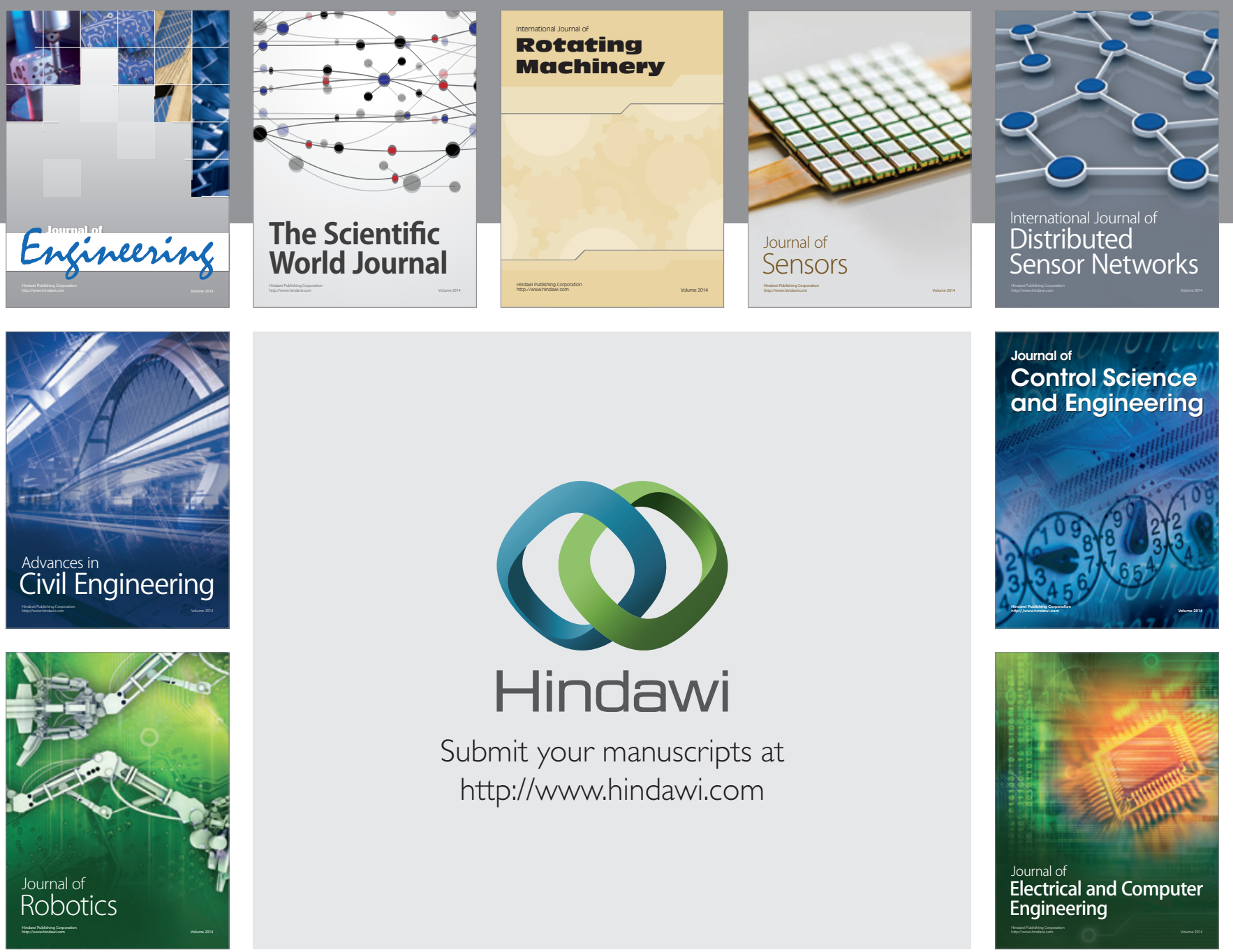

Submit your manuscripts at

http://www.hindawi.com
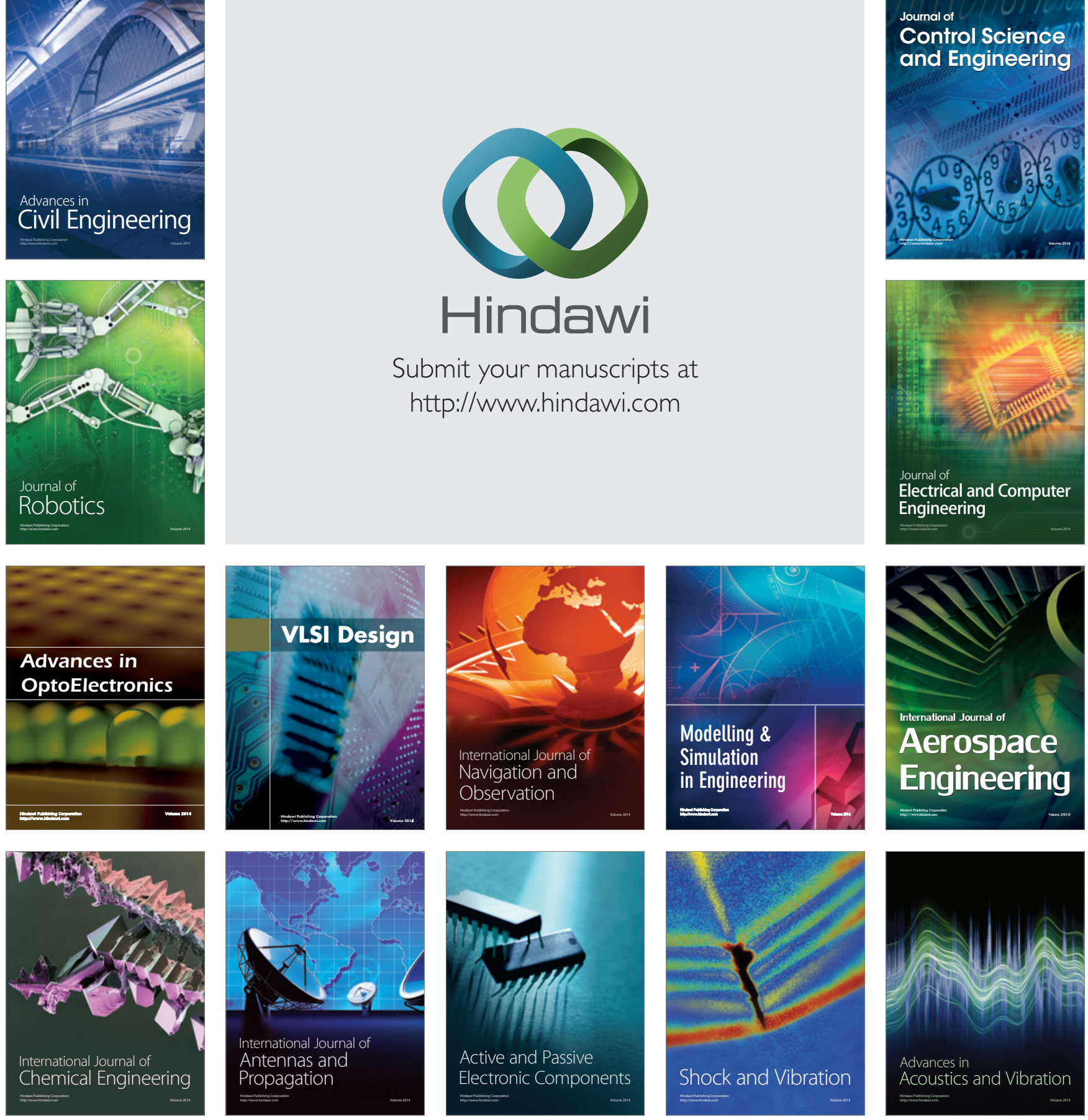